\title{
BMJ Open Mid-German Sepsis Cohort (MSC): a prospective observational study of sepsis survivorship
}

Carolin Fleischmann-Struzek (1) , ${ }^{1}$ Miriam Kesselmeier (1) , ${ }^{1,2,3}$ Dominique Ouart, ${ }^{1,4}$ Christiane S. Hartog (D) , ${ }^{1,4,5,6}$ Michael Bauer, ${ }^{1,4}$ Sven Bercker, ${ }^{7}$ Michael Bucher, ${ }^{8}$ Andreas Meier-Hellmann, ${ }^{9}$ Sirak Petros (D) , ${ }^{10}$ Torsten Schreiber, ${ }^{11}$ Philipp Simon (1D , ${ }^{7}$ Lorenz Weidhase, ${ }^{10}$ Sebastian Born (1D , ${ }^{1}$ Anke Braune, ${ }^{1,12}$ Hicham Chkirni, ${ }^{1,12}$ Cornelia Eichhorn, ${ }^{1,12}$ Sandra Fiedler, ${ }^{1,12}$ Christin Gampe, ${ }^{1,12}$ Christian König (D) , ${ }^{1,4}$ Stephanie Platzer, ${ }^{1,12}$ Heike Romeike, ${ }^{1}$ Kristin Töpfer, ${ }^{1}$ Konrad Reinhart, ${ }^{1,5}$ André Scherag (D) 1,2,3,12

To cite: Fleischmann-Struzek C, Kesselmeier M, Ouart D, et al. Mid-German Sepsis Cohort (MSC): a prospective observational study of sepsis survivorship. BMJ Open 2021;11:e043352. doi:10.1136/ bmjopen-2020-043352

- Prepublication history and additional materials for this paper are available online. To view these files, please visit the journal online (http://dx.doi. org/10.1136/bmjopen-2020043352).

Received 01 September 2020 Revised 26 January 2021 Accepted 04 February 2021

\section{Check for updates}

(c) Author(s) (or their employer(s)) 2021. Re-use permitted under CC BY-NC. No commercial re-use. See rights and permissions. Published by BMJ.

For numbered affiliations see end of article.

\section{Correspondence to} Professor André Scherag; andre.scherag@med.uni-jena. de

\section{ABSTRACT}

Purpose The Mid-German Sepsis Cohort (MSC) aims to investigate mid-term and long-term functional disabilities in sepsis survivors from intensive care unit (ICU) discharge until 1 year after. Secondary, post-acute mortality and morbidity, health-related quality of life and healthcare utilisation will be investigated.

Participants The MSC comprises adult (aged $\geq 18$ years) patients who were treated for (severe) sepsis or septic shock on ICU. The participants were recruited between 15 April 2016 and 30 November 2018 from five German centres. Three thousand two hundred and ten patients with sepsis were identified, of which 1968 survived their ICU stay and were eligible for enrolment in the follow-up cohort. Informed consent for follow-up assessment was provided by 907 patients ( $46.1 \%$ of eligible patients). Findings to date The recruitment of the participants for follow-up assessments and the baseline data collection is completed. Incidence of sepsis was 116.7 patients per 1000 ICU patients. In this cohort profile, we provide an overview of the demographics and the clinical characteristics of both the overall sepsis cohort and the ICU survivors who provided informed consent for follow-up assessment (907 out of 1968 ICU survivors (46.1\%)).

Future plans The follow-ups are conducted 3, 6 and 12 months after ICU discharge. Another yearly follow-up up to 5 years after ICU discharge is pursued. Several cooperation and satellite projects were initiated. This prospective cohort offers a unique resource for research on long-term sequelae of sepsis survivors.

Trial registration number German Clinical Trials Registry (DRKS00010050).

\section{INTRODUCTION}

Sepsis, defined as a dysregulated host response to infection leading to life-threatening organ dysfunction, is a medical emergency which requires rapid and adequate treatment. ${ }^{1}$ In 2017, an estimated 48.9 million sepsis cases were recorded worldwide, of which approximately 37.9 million patients survived the acute

\section{Strengths and limitations of this study}

- The Mid-German Sepsis Cohort (MSC) is one of the largest prospective sepsis cohorts to date and is special due to the wide spectrum of morbidities and functional outcomes assessed.

- Consecutive enrolment of all adult patients with sepsis was realised by daily screening of all intensive care unit (ICU) patients by trained study nurses or physicians over the study period in five study centres.

- Comprehensive follow-up assessments are conducted 3, 6 and 12 months after ICU discharge.

- A potential selection bias towards younger and healthier participants consenting in the follow-up cannot be ruled out.

- Three of five participating sites were academic centres, which may account for the inclusion of patients with a higher severity of the disease.

care hospitalisation. ${ }^{2}$ However, sepsis is not overcome when patients are discharged from the hospital. In addition to increased late mortality, a majority of sepsis survivors suffer from life-changing long-term consequences. ${ }^{3}$ The acute disease can affect every organ system and pathway by mechanisms which are insufficiently understood, including inflammation, ischaemia and ischaemia reperfusion. Moreover, long-term consequences are exacerbated by use of invasive measures, drugs and prolonged immobilisation. Survivorship is associated with immunosuppression, ${ }^{4}$ inflammation-associated encephalopathy, ${ }^{5}$ damage to muscles and nerves (critical illness polyneuropathy and polymyopathy) acquired on intensive care units (ICUs) ${ }^{6}$ as well as anxiety and depression. ${ }^{7}$ Many survivors suffer from a co-occurrence of symptoms. ${ }^{8}$ 
Given this considerable health burden, the WHO emphasised the improvement of sepsis aftercare as a major priority in a recent resolution. ${ }^{9}$ However, existing research on sepsis survivorship is limited due to variable inclusion/exclusion criteria, outcome measures and timing of outcome assessments as well as analyses of small or highly selected patient populations with a focus on single domains. ${ }^{3}$ Thus, it is difficult to integrate and generalise the existing evidence. We need to know more about the incidence, extent, progression and co-occurrence of long-term consequences after sepsis in order to identify vulnerable patient groups and draw implications for appropriate aftercare and rehabilitation.

The Mid-German Sepsis Cohort (MSC) was set up to assess long-term morbidity after sepsis by a comprehensive follow-up of 3000 consecutive patients with sepsis recruited from ICUs in five participating hospitals in Germany. The primary outcome is functional disability as assessed by (instrumental) activities of daily living from ICU discharge to 1 year after. Secondary outcomes comprise long-term mortality and morbidity, healthrelated quality of life and healthcare utilisation. A study protocol has been previously published. ${ }^{10}$ This cohort profile reports on the baseline characteristics of the recruited patients during their (index) ICU/hospital stay.

\section{COHORT DESCRIPTION \\ Recruitment}

The MSC is a prospective observational study, for which patient recruitment took place between 15 April 2016 and 30 November 2018 in the ICUs of five German hospitals. Per protocol, an additional ICU in the acute care hospital and rehabilitation centre Kreischa was planned for recruitment, but withdrew their participation prior to study beginning. Basic description of the participating centres is provided in online supplemental table 1. All ICU patients treated in the participating centres were screened daily for eligibility. Patients were eligible if they were aged $\geq 18$ years (at ICU discharge), were diagnosed with (severe) sepsis or septic shock and had no prior enrolment in the MSC. (Severe) sepsis is defined as clinically suspected or microbiologically proven infection and presence of at least one organ dysfunction due to infection. Septic shock is defined as persistent infectionrelated hypotension (systolic arterial blood pressure $\leq 90$ $\mathrm{mm} \mathrm{Hg}$ or mean arterial blood pressure $\leq 65 \mathrm{~mm} \mathrm{Hg}$ for $>1$ hour, or need of vasopressor support to raise the blood pressure above these limits; online supplemental table 2). We applied the organ dysfunction and shock definitions of the German Sepsis Society valid between 2016 and $2018,{ }^{11}$ which are in accordance with the sepsis-1 criteria. Documentation of the study also included lactate levels and the Sequential (sepsis-related) Organ Failure Assessment (SOFA) score, so that the sepsis- 3 criteria $^{1}$ could also be applied retrospectively. According to the institutional review boards (IRBs, including data protection/ privacy aspects), informed consent was not necessary to obtain routinely documented patient data according to the federal hospital laws of Thuringia, Saxony and Saxony-Anhalt for our research purpose, but was required for follow-up investigations with patients and proxies. In order to obtain written informed consent for follow-up, patients or legal representatives were approached in the hospital. If neither patient nor legal representative was available, a family member or close relative was asked for permission to contact the patient after hospital discharge in order to obtain consent. In this case, consent was asked in the first follow-up assessment. Accordingly, all consecutive patients with sepsis who were treated in the ICU constitute the 'ICU sepsis sample', while all ICU survivors who gave written informed consent and have sufficient German language skills were included in the follow-up assessments and constitute the 'follow-up sepsis sample'. For the arrangement of the follow-up appointments, patients were contacted by phone (twice) or mail (once), that is, in sum up to three times for each appointment. If patients missed two subsequent follow-up assessments (no contact or denial), they were deemed 'lost to follow-up'.

\section{Measurements and data collection}

Before the start of patient screening, all centre representatives met in person and a consensus was reached on the data to be collected. After 12 months, a protocol amendment was submitted to and endorsed by IRBs to allow for a more detailed documentation regarding delirium. The documentation of hospital baseline data and follow-up interviews is performed via web-based electronic case report forms in the validated management software 'OpenClinica' by trained study nurses, physicians or experienced and trained medical students.

Hospital (baseline) data were documented in the centres at sepsis onset in the ICU, at ICU discharge and at hospital discharge. Sepsis onset was defined as time of ICU admission for patients admitted with sepsis or as the respective time point during the ICU stay. We assessed underlying infection focus, onset (hospital-acquired vs community-acquired $)^{12}$ and sepsis-related organ dysfunctions (online supplemental table 2). Furthermore, laboratory values (eg, lactate, creatinine, white cell count), diagnostic measures (eg, blood culture sampling), therapies (eg, mechanical ventilation, vasopressor therapy), documented pre-existing comorbidities according to the Charlson Comorbidity Index,${ }^{13}$ the maximum SOFA score, end-of-life decisions (do not resuscitate, withhold, withdraw therapy order), length of ICU/hospital stay and time/cause of death were taken from the electronic medical records. We also collected data on presence and length of delirium as identified by specific scores at use in the participating centres (positive Confusion Assessment Method for the ICU, Intensive Care Delirium Screening Checklist $\geq 4$, Nursing Delirium Screening Scale $\geq 2$ ) or clinical judgement of the treating physicians. If information on single comorbidities, organ dysfunctions, systemic inflammatory response syndrome criteria or infection 


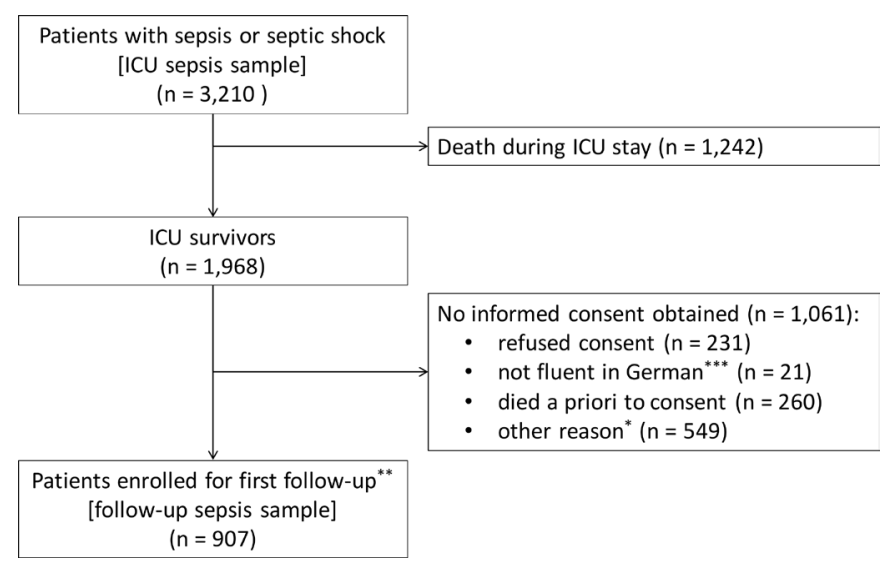

Figure 1 Patient flow of the follow-up sepsis sample of the Mid-German Sepsis Cohort. *Includes primarily patients who were discharged to hospice and thus were not further contacted; or patients who did not reply within 3 months after ICU discharge (for details on the standardised contact procedure, see text). ${ }^{\star \star}$ Includes the permission provided by a close relative or spouse to contact the patient for later consent (after hospital discharge). ${ }^{\star * \star}$ Given that follow-up interviews were performed in German. ICU, intensive care unit; n, number.

foci was not documented, they were considered as not pre-existing or present.

The follow-up interviews are scheduled 3, 6 and 12 months after ICU discharge and yearly thereafter. They are performed centrally by the Jena University Hospital study team. Follow-up assessment includes physical, functional, cognitive and mental health outcomes. Furthermore, sociodemographic information, dependence for chronic care, hospital readmissions, recurrence of sepsis or severe infections, use of rehabilitation or ambulant therapies, and a list of potential sepsis sequelae in patient-reported terms were assessed from the patients, patients' relatives or caregivers. Interviews are performed by telephone or face-to-face. Face-to-face interviews were offered to patients irrespective from which centre they were recruited. For further details, we refer to the study protocol. $^{10}$

\section{Characteristics of study participants}

Patient flow from sepsis onset to follow-up enrolment is provided in figure 1 . Three thousand two hundred and ten patients were identified with sepsis and constitute the ICU sepsis sample. Among the 1968 ICU survivors, 907 patients $(46.1 \%)$ provided consent and constitute the follow-up sepsis sample. Baseline characteristics of the patients are provided in table 1 . The consent rate was lower in patients with pre-existing dementia $(21.5 \%)$, but did not differ in scale in patients with pre-existing severe liver disease $(48.9 \%)$, congestive heart failure $(46.1 \%)$ or patients discharged with mechanical ventilation from the hospital (44.1\%).

A comparison of all ICU survivors who provided informed consent (follow-up sepsis sample) and those who did not is provided in online supplemental table 3 .
Patients in the follow-up sample were younger and less comorbid, including a lower proportion of patients who suffered from dementia. They less often received mechanical ventilation or therapeutic limitations (indicating a palliative care indication) and had a lower maximal SOFA score and a shorter hospital length of stay. Furthermore, there were differences in the distributions of the kind of organ dysfunctions and the infection foci between ICU survivors with and without informed consent. However, we did not observe differences in the frequency of septic shock.

\section{FINDINGS TO DATE}

Among 3210 ICU-treated patients with sepsis, ICU mortality was $38.7 \%$ and hospital mortality was $47.4 \%$. In-hospital survival rates are provided in figure 2. Three out of five participating hospitals ensured a continuous daily patient screening over the study period. Based on these three study centres, which were academic centres, the overall incidence of sepsis was 116.7 patients per 1000 ICU patients. This is in the range of the incidence and mortality estimates from two previous prospective studies which assessed ICU-treated patients with sepsis using comparable sepsis criteria in Germany. In 2007, Engel et $a l^{4}$ identified 415 patients $(10.7 \%)$ with sepsis among 3877 ICU patients with a hospital mortality of $55.2 \%$. In the nationwide point prevalence study INSEP, ${ }^{15} 1503$ patients (12.6\%) with sepsis were identified out of 11883 ICU patients with a hospital mortality of $40.4 \%$ in 2016 . In a population-based analysis of ICU-treated and non-ICUtreated patients with sepsis based on hospital discharge data in Germany, the estimated hospital mortality was $41.7 \% .^{16}$

Of note, the ICU sepsis sample is one of the largest prospective sepsis cohorts to date. Most of the other prospective sepsis studies with clinical documentation were no cohort studies or did not realise a sequential patient screening/inclusion. Examples are a Brazilian point-prevalence study (794 patients with sepsis among 2632 ICU patients in 2014, hospital mortality of $55.7 \%),{ }^{17}$ the worldwide point-prevalence audit ICON (2973 patients with sepsis of 10069 ICU patients, hospital mortality of $35.3 \%),{ }^{18}$ a Japanese prospective sepsis registry (624 sepsis cases among 14417 ICU patients, hospital mortality $29.5 \%)^{19}$ or the PROGRESS registry (14543 patients with sepsis, hospital mortality of $49.7 \%) .^{20}$

The follow-up sepsis sample of 907 patients is one of the largest. Previously, long-term follow-up studies included considerably fewer patients, for example, Marra et al $\ell^{8}$ (259 patients with sepsis), Biason $e t a l^{21}$ (242 patients with sepsis) or Battle et a $t^{22}$ (106 patients with sepsis).

Given the difficulty of obtaining written informed consent from severely ill patients, stressed relatives or from discharged survivors (see also online supplemental table 3 ), we believe that the proportion of included patients $(46.1 \%)$ is still satisfying and compares favourably with those from other follow-up cohort studies of ICU patients. 
Table 1 Demographic and clinical characteristics of patients with sepsis with respect to their enrolment in the intensive care unit (ICU) and follow-up sepsis sample

\begin{tabular}{|c|c|c|c|c|}
\hline \multirow[b]{2}{*}{ Characteristic } & \multicolumn{2}{|c|}{$\begin{array}{l}\text { ICU sepsis sample } \\
(\mathrm{n}=3210)\end{array}$} & \multicolumn{2}{|c|}{$\begin{array}{l}\text { Follow-up sepsis sample } \\
(\mathrm{n}=907)\end{array}$} \\
\hline & $\mathbf{N}$ & Distribution & $\mathbf{N}$ & Distribution \\
\hline Age, in years & 3210 & $67(58-77)$ & 907 & $65(56-74)$ \\
\hline Male sex & 3210 & 2054 (64.0\%) & 907 & $584(64.4 \%)$ \\
\hline Comorbidities $^{*}$ as documented in the patient file & 3206 & & 906 & \\
\hline Diabetes & & 957 (29.9\%) & & $265(29.2 \%)$ \\
\hline Chronic pulmonary disease & & $535(16.7 \%)$ & & $153(16.9 \%)$ \\
\hline Renal disease & & $458(14.3 \%)$ & & $110(12.1 \%)$ \\
\hline Congestive heart failure and myocardial infarction & & $753(23.5 \%)$ & & $191(21.1 \%)$ \\
\hline Cancer & & $837(26.1 \%)$ & & $216(23.8 \%)$ \\
\hline Dementia & & $144(4.5 \%)$ & & $20(2.2 \%)$ \\
\hline Cerebrovascular disease & & $256(8.0 \%)$ & & $65(7.2 \%)$ \\
\hline Liver disease & & $362(11.3 \%)$ & & $75(8.3 \%)$ \\
\hline HIV/AIDS & & $7(0.2 \%)$ & & $5(0.6 \%)$ \\
\hline Other & & $534(16.7 \%)$ & & $121(13.4 \%)$ \\
\hline Number of comorbidities & & $1(1-2)$ & & $1(1-2)$ \\
\hline \multicolumn{5}{|l|}{ Distribution } \\
\hline 0 & & $631(19.7 \%)$ & & $212(23.4 \%)$ \\
\hline 1 & & $1114(34.7 \%)$ & & $339(37.4 \%)$ \\
\hline $2-4$ & & $1373(42.8 \%)$ & & $333(36.8 \%)$ \\
\hline$>4$ & & $88(2.7 \%)$ & & $22(2.4 \%)$ \\
\hline Charlson Comorbidity Index & & $4(3-6)$ & & $4(2-6)$ \\
\hline Admission type & 3210 & & 907 & \\
\hline Non-surgical emergency & & $2367(73.7 \%)$ & & $647(71.3 \%)$ \\
\hline Surgical emergency & & $597(18.6 \%)$ & & $185(20.4 \%)$ \\
\hline Elective surgery & & $246(7.7 \%)$ & & $75(8.3 \%)$ \\
\hline $\begin{array}{l}\text { Incidence of sepsis/septic shock, in patients per } \\
1000 \text { ICU patients } †\end{array}$ & & 116.7 & & \\
\hline Origin of infection & 3210 & & 907 & \\
\hline Hospital-acquired & & $1754(54.6 \%)$ & & $476(52.5 \%)$ \\
\hline Community-acquired & & $1456(45.4 \%)$ & & $431(47.5 \%)$ \\
\hline Focus of infection & 3209 & & 906 & \\
\hline Known & & $2966(92.4 \%)$ & & $843(93.0 \%)$ \\
\hline \multicolumn{5}{|l|}{ Among them: } \\
\hline Pneumonia & & $1473(49.7 \%)$ & & $379(45.0 \%)$ \\
\hline Other upper or lower respiratory tract & & $216(7.3 \%)$ & & $59(7.0 \%)$ \\
\hline Intra-abdominal & & $577(19.5 \%)$ & & $195(23.1 \%)$ \\
\hline Primary bacteraemia & & $533(18.0 \%)$ & & $122(14.5 \%)$ \\
\hline Urogenital & & $439(14.8 \%)$ & & $117(13.9 \%)$ \\
\hline Bones/soft tissue & & $280(9.4 \%)$ & & $82(9.7 \%)$ \\
\hline Postoperative wound infection & & $135(4.6 \%)$ & & $27(3.2 \%)$ \\
\hline Gastrointestinal & & $133(4.5 \%)$ & & $28(3.3 \%)$ \\
\hline Thoracic (empyema/mediastinitis) & & $89(3.0 \%)$ & & $36(4.3 \%)$ \\
\hline Cardiovascular & & $86(2.9 \%)$ & & $26(3.1 \%)$ \\
\hline Device-related infection & & $79(2.7 \%)$ & & $23(2.7 \%)$ \\
\hline
\end{tabular}


Table 1 Continued

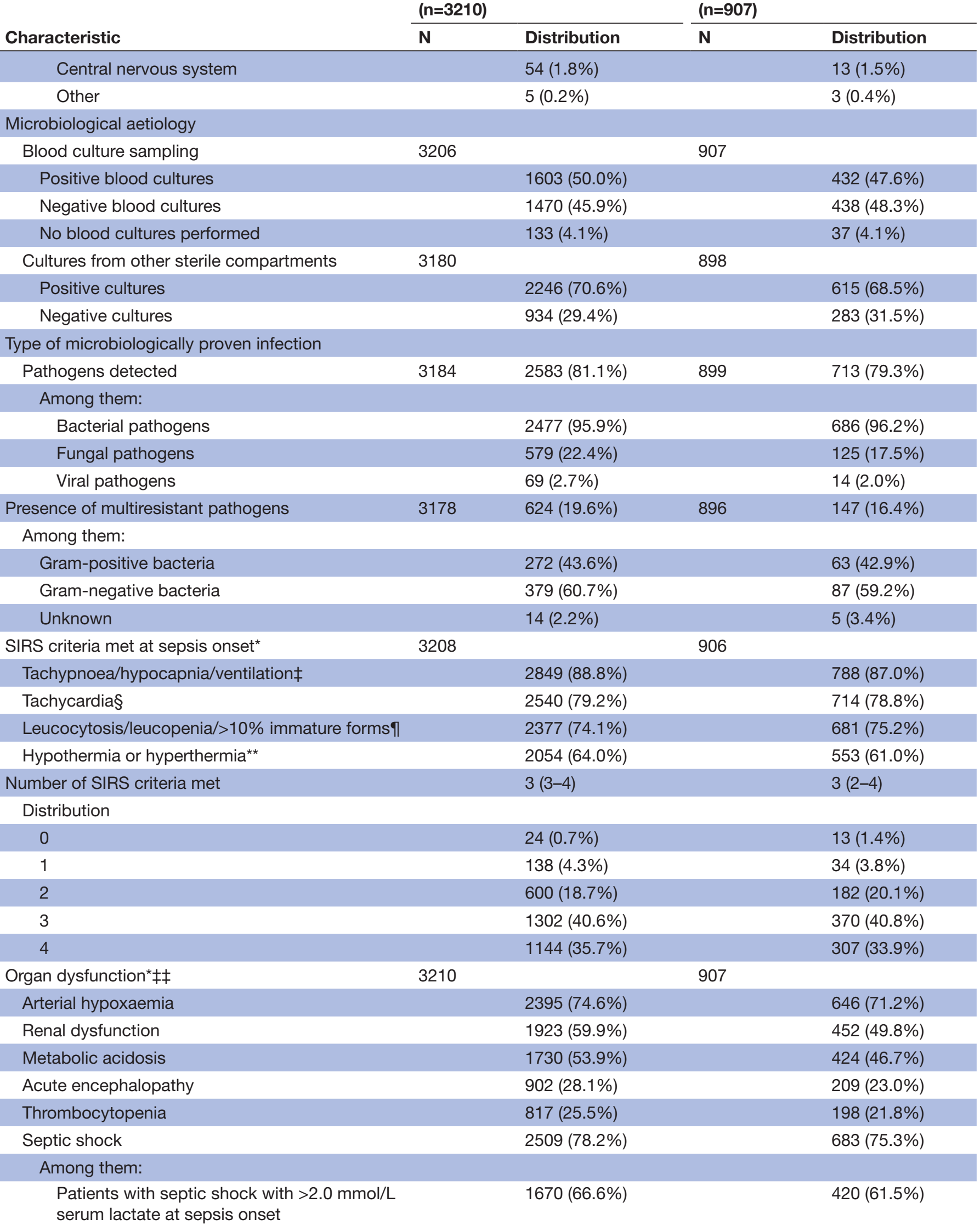


Table 1 Continued

\begin{tabular}{|c|c|c|c|c|}
\hline \multirow[b]{2}{*}{ Characteristic } & \multicolumn{2}{|c|}{$\begin{array}{l}\text { ICU sepsis sample } \\
(n=3210)\end{array}$} & \multicolumn{2}{|c|}{$\begin{array}{l}\text { Follow-up sepsis sample } \\
(\mathrm{n}=907)\end{array}$} \\
\hline & $\mathbf{N}$ & Distribution & $\mathbf{N}$ & Distribution \\
\hline Number of organ dysfunctions & & $3(2-4)$ & & $3(2-4)$ \\
\hline \multicolumn{5}{|l|}{ Distribution } \\
\hline 0 & & $0(0.0 \%)$ & & $0(0.0 \%)$ \\
\hline 1 & & $292(9.1 \%)$ & & $116(12.8 \%)$ \\
\hline 2 & & $702(21.9 \%)$ & & $246(27.1 \%)$ \\
\hline$>2$ & & $2216(69.0 \%)$ & & $545(60.1 \%)$ \\
\hline Presence of delirium during ICU stay & 3201 & $1062(33.2 \%)$ & 906 & $288(31.8 \%)$ \\
\hline Duration in respective patients, in days & & $4(2-8)$ & & $4(2-9)$ \\
\hline Vasopressor therapy during ICU stay & 3208 & $2738(85.3 \%)$ & 907 & $721(79.5 \%)$ \\
\hline \multicolumn{5}{|c|}{ Organ replacement or support therapy during ICU stay } \\
\hline Mechanical ventilation & 3205 & 2587 (80.7\%) & 905 & 627 (69.3\%) \\
\hline \multicolumn{5}{|l|}{ Among them: } \\
\hline Controlled ventilation & & $1378(53.3 \%)$ & & $340(54.2 \%)$ \\
\hline Duration in respective patients, in days & & $4(2-11)$ & & $6(2-16)$ \\
\hline Assisted ventilation & & $1597(61.7 \%)$ & & $327(52.2 \%)$ \\
\hline Duration in respective patients, in days & & $6(3-15)$ & & $8(2-21)$ \\
\hline ECMO or other lung replacement therapy & 3204 & $73(2.3 \%)$ & 906 & $17(1.9 \%)$ \\
\hline Duration in respective patients, in days & & $7(5-9)$ & & $9(6-14)$ \\
\hline Renal replacement therapy & 3200 & $1466(45.8 \%)$ & 901 & $273(30.3 \%)$ \\
\hline Other replacement therapy & 3202 & $22(0.7 \%)$ & 905 & $5(0.6 \%)$ \\
\hline Maximal SOFA score during ICU stay & 2911 & $15(12-18)$ & 815 & $13(10-15)$ \\
\hline Length of ICU stay, in days & 3210 & $9(4-21)$ & 907 & $10(4-26)$ \\
\hline Length of hospital stay, in days & 3210 & $25(13-43)$ & 907 & $34(21-52)$ \\
\hline \multicolumn{5}{|l|}{ ICU mortality } \\
\hline Overall & 3210 & $1242(38.7 \%)$ & & \\
\hline In patients with septic shock†† & 2509 & $1056(42.1 \%)$ & & \\
\hline In patients without septic shock †† & 632 & $166(26.3 \%)$ & & \\
\hline Cause of death among ICU decedents & 1242 & & & \\
\hline Sepsis as direct or indirect cause & & $1180(95.0 \%)$ & & \\
\hline Other causes of death & & $62(5.0 \%)$ & & \\
\hline \multicolumn{5}{|l|}{ Hospital mortality } \\
\hline Overall & 3210 & $1520(47.4 \%)$ & 907 & $61(6.7 \%)$ \\
\hline In patients with septic shock†† & 2509 & $1265(50.4 \%)$ & 683 & $46(6.7 \%)$ \\
\hline In patients without septic shock†† & 632 & $234(37.0 \%)$ & 198 & $15(7.6 \%)$ \\
\hline Cause of death among hospital decedents & 1519 & & 61 & \\
\hline Sepsis as direct or indirect cause & & $1400(92.2 \%)$ & & $45(73.8 \%)$ \\
\hline Other causes of death & & $119(7.8 \%)$ & & $16(26.2 \%)$ \\
\hline Limitation of life-sustaining therapy & 3188 & $1170(36.7 \%)$ & 900 & $49(5.4 \%)$ \\
\hline \multicolumn{5}{|l|}{ Among them: } \\
\hline DNR & & $838(71.6 \%)$ & & $44(89.8 \%)$ \\
\hline Withhold & & $560(47.9 \%)$ & & $13(26.5 \%)$ \\
\hline Withdraw & & $572(48.9 \%)$ & & $2(4.1 \%)$ \\
\hline Tracheostomy at hospital discharge & 1689 & $287(17.0 \%)$ & 845 & 129 (15.3\%) \\
\hline
\end{tabular}




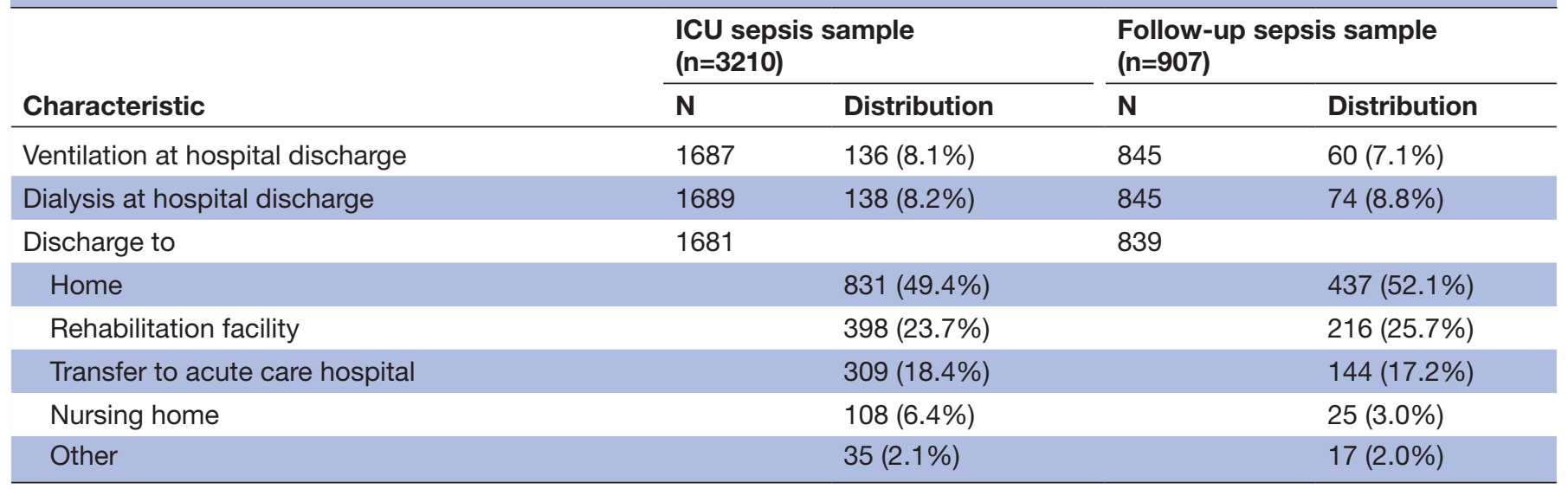

Absolute and relative frequencies or median with first and third quartile are provided (distribution). The number of patients in the respective sample (n) is indicated. For several characteristics, multiple answers per patient were possible. Note that numbers do not necessarily add up to the total number of patients due to missing/unknown values; number of patients with information in the respective item $(\mathrm{N})$ is provided in a separate column. Relative frequencies are related to these numbers if not otherwise indicated.

*If individual items were not documented, they were considered as not existent. Patients with no documentation were excluded.

†Approximation based on information in terms of ICU patients per year provided by the three (academic) study centres that ensured a continuous screening of ICU patients over the study period.

$\ddagger$ Tachypnoea ( $\geq 20$ breaths/min) and/or arterial partial pressure of carbon dioxide $\leq 4.3 \mathrm{kPa}(32 \mathrm{~mm} \mathrm{Hg})$ and/or mechanical ventilation.

$\S \geq 90$ beats/min.

|leucocytosis (leucocyte count $\geq 12 \times 10^{9} / \mathrm{L}$ ) or leucopenia (leucocyte count $\leq 4 \times 10^{9} / \mathrm{L}$ ) and/or $>10 \%$ immature forms.

${ }^{\star *}$ Hypothermia (body temperature $\leq 36^{\circ} \mathrm{C}$ ) or hyperthermia (body temperature $\geq 38^{\circ} \mathrm{C}$ ).

††Missing information on the presence of a septic shock: in 69 patients of the ICU and in 26 patients of the follow-up sepsis sample.

¥¥Definitions of these organ dysfunctions are provided in online supplemental table 2.

DNR, do not resuscitate; ECMO, extracorporeal membrane oxygenation; ICU, Intensive Care Unit; SIRS, systemic inflammatory response syndrome; SOFA, Sequential Organ Failure Assessment.

The multicentre cohort study in Germany of ICU patients with acute respiratory distress syndrome (DACAPO trial) included 876 patients of 1900 eligible patients $(46.1 \%)$ in the follow-up assessment. ${ }^{23}$ Pandharipande $e t a l^{24}$ enrolled in the BRAIN-ICU Study 826 patients (medical or surgical ICU patients with respiratory failure, cardiogenic shock

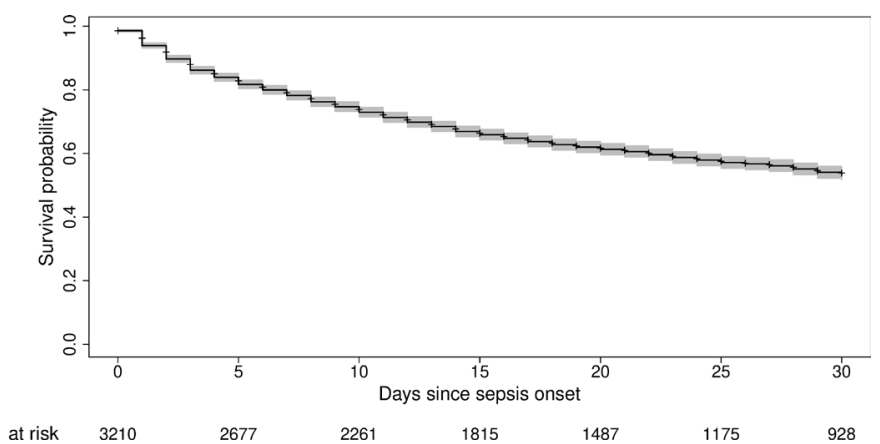

Figure 2 In-hospital survival since sepsis onset (in days) of patients in the ICU sepsis sample. The survival curve is censored at day 30 . The number of participants at risk is given below the plot area. Right censoring is indicated by + and $95 \%$ confidence intervals are provided in grey. ICU, intensive care unit. or septic shock) of 5210 eligible patients (15.9\%) in the USA. Mitchell et $a l^{25}$ enrolled 148 patients of 421 eligible general ICU patients (35.2\%) in Australia to assess longterm cognitive impairment and delirium among survivors from critical illness.

\section{STRENGTHS AND LIMITATIONS}

The MSC has several strengths, including the prospective study design with its focus on post-ICU assessment and measures to ensure consistent data quality. The latter comprises, for example, onsite training and monitoring in all participating centres, use of a good clinical practice conform internet-based database comprising an integrated audit trail and electronic plausibility checks, daily screening of all ICU patients by trained study nurses or physicians over the study period and the consecutive enrolment of all patients aged 18 years or older. Sepsis surveillance and follow-up in prospective cohorts is therefore considered advantageous compared with administrative data. ${ }^{26}$ The MSC is also special due to the wide spectrum of morbidities assessed including cognitive dysfunction, post-traumatic stress symptoms, depression, 
fatigue or pain (for details see the previously published protocol).${ }^{10}$ By this, we aim to gain important and valid insights into the (long-term) burden and dynamics of post-sepsis morbidity of ICU-treated patients with sepsis. However, the study also has several limitations. Three of five participating sites were academic centres, which may account for the inclusion of patients with a higher severity of the disease. These centres implemented a continuous screening over the 2.5-year study period and contributed data to the analysis of sepsis incidence among ICUtreated patients. The remaining two centres also realised a complete screening of ICU-treated patients but only for a shorter or a discontinuous period of time. Their patients were also eligible for follow-up, but their data were not included in the incidence estimation. Furthermore, our cohort is restricted to ICU patients from selected hospitals, and thereby does not capture information on the considerable proportion of patients with sepsis treated outside the ICU (46.2\% in 2015 according to nationwide hospital discharge data) ${ }^{16}$ or patients with sepsis in the emergency department. Changing ICU admission policies and capacities over time and in different regions/ countries also limits the comparability and conclusion about the general population of ICU populations. ${ }^{26}$ Moreover, there was a certain proportion of ICU survivors who we could not reach to obtain informed consent, which may have introduced a certain selection bias towards younger and healthier patients included in the follow-up sepsis sample. This may lead to an underestimation of long-term sequelae, which affect older and pre-morbid patients more frequently. ${ }^{27}$ In addition, about $10 \%$ of eligible ICU survivors or their proxies refused consent. Obtaining consent for observational studies in critically ill patients and their proxies remains challenging. Nevertheless, a participation rate of about $46.1 \%$ is higher than in other cohorts.

\section{COLLABORATIONS}

The MSC is linked to the ICROS Study ${ }^{28}$ which has a focus on deep phenotyping including clinical and laboratory tests, cardiovascular function, metabolome, lipidome, microbiome, mitochondrial oxygen metabolism, heart rate variability, body composition and immune status assessments. Both studies (MSC and ICROS) ${ }^{10} 28$ use the same core documentation. However, the ICROS Study is a monocentric study that aims at including three cohorts: 130 patients with sepsis, 80 patients with cardiomyopathy without infection and 80 healthy individuals. The overlap of the studies enables analyses that include both studies. Furthermore, the MSC has already served to support several add-on projects such as a comparative validation of three screening instruments to assess symptoms of post-traumatic stress disorder after intensive care for sepsis. ${ }^{29}$ Patients enrolled in the MSC were also invited to participate in another interview study on satisfaction with follow-up care and rehabilitation after sepsis, which forms part of the SEPFROK Study (Sepsis long-term impairments, risk factors, healthcare use and costs study; German Clinical Trials Registry number DRKS00016340). For details regarding the availability of data for potential new collaborators, see the data sharing section.

\section{Author affiliations}

${ }^{1}$ Integrated Research and Treatment Center, Center for Sepsis Control and Care (CSCC), Jena University Hospital, Jena, Germany

${ }^{2}$ Research Group Clinical Epidemiology, CSCC, Jena University Hospital, Jena, Germany

${ }^{3}$ Institute of Medical Statistics, Computer and Data Sciences, Jena University Hospital, Jena, Germany

${ }^{4}$ Department of Anesthesiology and Intensive Care Medicine, Jena University Hospital, Jena, Germany

${ }^{5}$ Department of Anesthesiology and Intensive Care Medicine, Charité -

Universitätsmedizin Berlin, Berlin, Germany

${ }^{6}$ Klinik Bavaria Kreischa, Kreischa, Germany

${ }^{7}$ Department of Anaesthesiology and Intensive Care Medicine, University Hospital Leipzig, Leipzig, Germany

${ }^{8}$ Department of Anesthesiology and Critical Care Medicine, Halle-Wittenberg University, Halle, Germany

${ }^{9}$ Helios-Kliniken, Berlin, Berlin, Germany

${ }^{10}$ Medical Intensive Care Unit, University Hospital Leipzig, Leipzig, Germany

${ }^{11}$ Zentrum für Anästhesie, Intensivmedizin und Notfallmedizin, Zentralklinik Bad

Berka GmbH, Bad Berka, Germany

${ }^{12}$ Center for Clinical Studies, Jena University Hospital, Jena, Germany

\section{Twitter André Scherag @ScheragAndre}

Acknowledgements The authors would like to thank the participating patients and their relatives for their contribution to clinical sepsis research. Furthermore, we thankfully acknowledge the strong commitment of study nurses and physicians in the participating centres of the Mid-German Sepsis Cohort. We thank Wes Ely for thoughtful comments and suggestions during the planning stage.

Contributors CF-S, MK and DO contributed equally to this work. AS and KR designed the study and applied for funding. AS, KR, CSH, CF-S and DO drafted the clinical research forms. MBa, SBe, MBu, AM-H, SP, TS, PS and LW coordinated the conduction of the study and data acquisition at the participating study centres. $\mathrm{HC}, \mathrm{SF}$ and SP were responsible for the project management of the study. CE was responsible for the data management in OpenClinica. AB and CG performed the monitoring of the study sites. HR, KT, CK, DO and CF-S performed follow-up interviews. MK performed the statistical analyses under the supervision of AS. AS, CSH, MK, DO, CF-S, SBo and KR interpreted the data. AS, CSH, MK, DO and CF-S drafted the manuscript. All authors critically revised the manuscript and approved the final version.

Funding This work was supported by the Integrated Research and Treatment Center, Center for Sepsis Control and Care (CSCC) at the Jena University Hospital funded by the German Ministry of Education and Research (BMBF No. 01 E01002 and 01E01502) and by the Rudolf Presl GmbH \& Co, Kreischa, Germany.

Disclaimer The funders have/had no role in study design, data collection and analysis, decision to publish or preparation of this and future manuscripts.

Competing interests CSH declares funding from the German Federal Joint Committee Innovations-Funds and the European Society of Intensive Care Medicine. All other authors declare no conflicts of interest.

Patient consent for publication Not required.

Ethics approval The study was approved by four local/federal responsible institutional ethics committees (lead: IRB of the Jena University Hospital, no. 466901/16) and by the respective federal data protection commissioners.

Provenance and peer review Not commissioned; externally peer reviewed.

Data availability statement Data are available upon reasonable request. Data access to the final cleaned data set is provided to all project applicants (primarily within the MSC investigator group) along with written use and access rules of the CSCC which include a brief proposal including a sketch indicating the envisaged analysis project and an additional ethical or data protection vote depending on the type of project. To ensure confidentiality, data distributed to project applicants will be double pseudonymised and any directly identifying patient information will not be provided. Due to data economy and subsequently 
data protection, only the variables required for the analysis project will be provided.

Supplemental material This content has been supplied by the author(s). It has not been vetted by BMJ Publishing Group Limited (BMJ) and may not have been peer-reviewed. Any opinions or recommendations discussed are solely those of the author(s) and are not endorsed by BMJ. BMJ disclaims all liability and responsibility arising from any reliance placed on the content. Where the content includes any translated material, BMJ does not warrant the accuracy and reliability of the translations (including but not limited to local regulations, clinical guidelines, terminology, drug names and drug dosages), and is not responsible for any error and/or omissions arising from translation and adaptation or otherwise.

Open access This is an open access article distributed in accordance with the Creative Commons Attribution Non Commercial (CC BY-NC 4.0) license, which permits others to distribute, remix, adapt, build upon this work non-commercially, and license their derivative works on different terms, provided the original work is properly cited, appropriate credit is given, any changes made indicated, and the use is non-commercial. See: http://creativecommons.org/licenses/by-nc/4.0/.

\section{ORCID iDs}

Carolin Fleischmann-Struzek http://orcid.org/0000-0002-1104-3191

Miriam Kesselmeier http://orcid.org/0000-0001-6462-2579

Christiane S. Hartog http://orcid.org/0000-0003-1098-9775

Sirak Petros http://orcid.org/0000-0002-2345-756x

Philipp Simon http://orcid.org/0000-0003-2696-3254

Sebastian Born http://orcid.org/0000-0001-5803-7963

Christian König http://orcid.org/0000-0001-7671-231X

André Scherag http://orcid.org/0000-0002-9406-4704

\section{REFERENCES}

1 Singer M, Deutschman CS, Seymour CW, et al. The third International consensus definitions for sepsis and septic shock (Sepsis-3). JAMA 2016;315:801-10.

2 Rudd KE, Johnson SC, Agesa KM, et al. Global, regional, and national sepsis incidence and mortality, 1990-2017: analysis for the global burden of disease study. Lancet 2020;395:200-11.

3 Prescott HC, Iwashyna TJ, Blackwood B, et al. Understanding and enhancing sepsis survivorship. priorities for research and practice. Am J Respir Crit Care Med 2019;200:972-81.

4 Hotchkiss RS, Monneret G, Payen D. Sepsis-induced immunosuppression: from cellular dysfunctions to immunotherapy. Nat Rev Immunol 2013;13:862-74.

5 Barichello T, Sayana P, Giridharan VV, et al. Long-term cognitive outcomes after sepsis: a translational systematic review. Mol Neurobiol 2019;56:186-251.

6 Zink W, Kollmar R, Schwab S. Critical illness polyneuropathy and myopathy in the intensive care unit. Nat Rev Neurol 2009;5:372-9.

7 Rosendahl J, Brunkhorst FM, Jaenichen D, et al. Physical and mental health in patients and spouses after intensive care of severe sepsis: a dyadic perspective on long-term sequelae testing the actor-partner interdependence model. Crit Care Med 2013;41:69-75.

8 Marra A, Pandharipande PP, Girard TD, et al. Co-occurrence of postintensive care syndrome problems among 406 survivors of critical illness. Crit Care Med 2018;46:1393-401.

9 World Health Organization Executive Board. Improving the prevention, diagnosis and management of sepsis, 2017.

10 Scherag A, Hartog CS, Fleischmann C, et al. A patient cohort on long-term sequelae of sepsis survivors: study protocol of the MidGerman sepsis cohort. BMJ Open 2017;7:e016827.
11 Reinhart K, Brunkhorst FM, Bone H-G. Prävention, diagnose, therapie und nachsorge der sepsis - erste revision der S2k-leitlinien der deutschen sepsis-gesellschaft e.V. (DSG) und der deutschen interdisziplinären vereinigung für intensiv- und notfallmedizin (DIVI). Anaesthesist 2010;59:347-70.

12 Nationales Referenzzentrum für Surveillance von nosokomialen Infektionen. Definitionen nosokomialer Infektionen (CDCDefinitionen). Berlin: Robert Koch-Institut, 2011.

13 Charlson ME, Pompei P, Ales KL, et al. A new method of classifying prognostic comorbidity in longitudinal studies: development and validation. J Chronic Dis 1987;40:373-83.

14 Engel C, Brunkhorst FM, Bone H-G, et al. Epidemiology of sepsis in Germany: results from a national prospective multicenter study. Intensive Care Med 2007;33:606-18.

15 SepNet Critical Care Trials Group. Incidence of severe sepsis and septic shock in German intensive care units: the prospective, multicentre INSEP study. Intensive Care Med 2016;42:1980-9.

16 Fleischmann-Struzek C, Mikolajetz A, Schwarzkopf D, et al. Challenges in assessing the burden of sepsis and understanding the inequalities of sepsis outcomes between National Health Systems: secular trends in sepsis and infection incidence and mortality in Germany. Intensive Care Med 2018;44:1826-35.

17 Machado FR, Cavalcanti AB, Bozza FA, et al. The epidemiology of sepsis in Brazilian intensive care units (the sepsis prevalence assessment database, SPREAD): an observational study. Lancet Infect Dis 2017;17:1180-9.

18 Vincent J-L, Marshall JC, Namendys-Silva SA, et al. Assessment of the worldwide burden of critical illness: the intensive care over nations (ICON) audit. Lancet Respir Med 2014;2:380-6.

19 Ogura H, Gando S, Saitoh D, et al. Epidemiology of severe sepsis in Japanese intensive care units: a prospective multicenter study. $J$ Infect Chemother 2014;20:157-62.

20 Martin G, Brunkhorst FM, Janes JM, et al. The International progress registry of patients with severe sepsis: drotrecogin alfa (activated) use and patient outcomes. Crit Care 2009;13:R103.

21 Biason L, Teixeira C, Haas JS, et al. Effects of sepsis on morbidity and mortality in critically ill patients 2 years after intensive care unit discharge. Am J Crit Care 2019;28:424-32.

22 Battle CE, Davies G, Evans PA. Long term health-related quality of life in survivors of sepsis in South West Wales: an epidemiological study. PLoS One 2014;9:e116304.

23 Dodoo-Schittko F, Brandstetter S, Brandl M, et al. Germanwide prospective DACAPO cohort of survivors of the acute respiratory distress syndrome (ARDS): a cohort profile. BMJ Open 2018;8:e019342.

24 Pandharipande PP, Girard TD, Jackson JC, et al. Long-term cognitive impairment after critical illness. N Engl J Med 2013;369:1306-16.

25 Mitchell ML, Shum DHK, Mihala G, et al. Long-term cognitive impairment and delirium in intensive care: a prospective cohort study. Aust Crit Care 2018;31:204-11.

26 Shappell CN, Klompas M, Rhee C. Surveillance strategies for tracking sepsis incidence and outcomes. J Infect Dis 2020;222:S74-83.

27 Iwashyna TJ, Ely EW, Smith DM, et al. Long-term cognitive impairment and functional disability among survivors of severe sepsis. JAMA 2010;304:1787-94.

28 Coldewey SM, Neu C, Baumbach P, et al. Identification of cardiovascular and molecular prognostic factors for the medium-term and long-term outcomes of sepsis (ICROS): protocol for a prospective monocentric cohort study. BMJ Open 2020;10:e036527.

29 Rosendahl J, Kisyova H, Gawlytta R, et al. Comparative validation of three screening instruments for posttraumatic stress disorder after intensive care. J Crit Care 2019;53:149-54. 